\title{
TRATAMIENTO ESTOMATOLÓGICO MULTIDISCIPLINARIO DE UN PACIENTE CON TRASTORNO DE ESPECTRO AUTISTA: REPORTE DE UN CASO
}

\section{MULTIDISCIPLINARY STOMATOLOGICAL TREATMENT OF A PATIENT WITH AUTISTIC SPECTRUM STORAGE: REPORT OF A CASE}

\author{
Alaniz Paredes Astrid ${ }^{1 *}$, Alvear-Córdova María Cristina ${ }^{2}$, Paredes Andrés ${ }^{3}$. \\ ${ }^{1}$ Universidad Nacional Autónoma de México. México \\ 2 Od. Esp en Odontopediatría. Universidad Católica de Cuenca. Ecuador. ${ }^{3}$ Od. Universidad Católica de Cuenca. Ecuador. \\ *astriday@hotmail.com
}

\begin{abstract}
Resumen
Introducción: El Trastorno de Espectro Autista (TEA) es un desorden neurológico que perdura por toda la vida, provocando alteraciones conductuales. Sus síntomas principales son las alteraciones en el comportamiento social, así como en la comunicación verbal y no verbal del niño. Objetivo: El propósito de este trabajo es enfatizar la importancia del apoyo multidisciplinario para el eficiente manejo estomatológico en los pacientes con TEA. Presentación del caso: Acude a la clínica de Odontopediatría un paciente masculino de 12 años de edad, con diagnóstico médico de Trastorno Autista, presentando un aumento de volumen en la zona retromolar inferior derecha, con patrón conductual Frankl 1 por lo que se decide hacer el tratamiento en un procedimiento bajo anestesia general. El manejo estomatológico fue realizado en tres tratamientos: 1. Tratamiento restaurador. 2. Tratamiento quirúrgico. 3. Tratamiento de control. Conclusiones: El profesional que brinde atención a pacientes con TEA tiene la misión de darles una mejor calidad de vida. Para esto, es indispensable conocer sus características y así determinar cómo se tratara a cada uno en base a su caso particular y al grado de severidad del trastorno.
\end{abstract}

Palabras clave: Anestesia general, Trastorno de Espectro Autista, Autismo.

\begin{abstract}
Introduction: Autism Spectrum Disorder (ASD) is a neurodevelopmental disorder that is life long and includes a wide range of behavioral disorders. The main symptoms of ASD are impairments in social interaction, verbal and nonverbal communication and the range of interests and activities of the child. Objective: The purpose of this report is to emphasize the importance of multidisciplinary work for the good stomatological management in patients with ASD. Case presentation: Attend clinic Pediatric Dentistry a male patient 12 years old with medical diagnosis of Autistic Disorder, showing an increase of volume in the lower right retromolar area with 1 Frankl's behavioral pattern is decided what to do treatment in a procedure by general anesthesia. The dental management was conducted in three treatments: 1 Restorative. 2. Surgical. 3. Control. Conclusions: The professional who provides care to patients with TEA's mission is to provide a better quality of life. For this, it is essential to understand its characteristics and determine how each were based on your particular case and the degree of severity of the disorder.
\end{abstract}

Key words: General Anesthetic, Autistic Disorder, Autism Spectrum.

\section{INTRODUCCIÓN}

Los Trastornos del Espectro Autista (TEA) constituyen una cadena de trastornos neuropsiquiátricos, tales como el trastorno de Asperger, Rett, desintegrativo infantil, entre otros estableciéndose como un problema de salud mental. ${ }^{1}$

El TEA se lo conceptualiza como un síndrome congé- nito degenerativo del Sistema Nervioso Central, así como el deterioro del crecimiento y del desarrollo cognitivo y social de quién la padece. ${ }^{2}$

Hay una diversidad de presentaciones de este síndrome, siendo muy diverso de un niño a otro, aunque la intensidad y frecuencia varían siempre se expresa una alteración en tres 
áreas primarias: ${ }^{3}$

1) Habilidades sociales.

2) Habilidades de lenguaje.

3) Comportamientos repetitivos y limitados. ${ }^{3}$

La prevalencia de TEA es de 5 por cada 10.000 niños, siendo cuatro veces más frecuente en los niños en comparación con las niñas. ${ }^{3}$

La complejidad de las manifestaciones clínicas que se presentan, sugieren la existencia de una etiología multicausal, los datos actuales apuntan hacia una etiología de influencia genética y medioambiental. ${ }^{4}$

También se relaciona como posibles causas a las complicaciones inmunológicas y obstétricas, así como las enfermedades tóxicas y opiáceos cerebrales exógenos. ${ }^{4}$

El comportamiento social inadecuado se considera el principal síntoma de los niños que sufren este trastorno, dado por la comunicación verbal y no verbal debido a los intereses y actividades exclusivas que el niño realiza. ${ }^{4}$

La interacción social en estos niños se caracteriza por las dificultades del lenguaje no verbal y esto se demuestra tanto en su posición corporal, así como en el contacto visual. El pobre desarrollo de las relaciones interpersonales y la falta de correspondencia social en las emociones son síntomas específicos de estos niños. ${ }^{5}$

La tardanza en el desarrollo del lenguaje evidentemente provoca alteración en la comunicación, la cual se ve más influenciada por el pobre lenguaje no verbal estereotipado que los niños poseen. Además, de las dificultades que se presentan al inicio y durante el desarrollo de las conversaciones, la escasa variedad del juego imaginativo y las limitaciones sociales completan el cuadro clínico de estos pacientes. ${ }^{5}$

La falta de interés en diversas actividades suelen expresarse como una hiperfocalización, sobre todo en los temas específicos, mientras que en los temas comunes o de rutina no funcionales existe una marcada cohesión del niño, dada por la existencia repetitiva de los estereotipos motores, al mismo tiempo que se observa una excesiva preocupación hacia los objetos. $^{5}$

Se ha determinado dos condiciones que se relacionan con el trastorno autista con mucha frecuencia: la epilepsia con un porcentaje que varía entre $20 \%$ al $30 \%$ de los casos y el trastorno por déficit de atención e hiperquinesia (TDAH) con un menor porcentaje, además de estas dos condiciones se ha observado que las alteraciones del sueño también están presentes en los niños que padecen este trastorno. ${ }^{6}$

Además de las alteraciones mencionadas se ha reportado alteraciones sensoperceptuales como hiper o hiposensibilidad a diversos estímulos ya sean estos auditivos, visuales o táctiles, siendo su percepción distorsionante de la integración multimodal. ${ }^{6}$

La conducta del paciente con trastorno autista presenta también una amplia gama de alteraciones. ${ }^{6}$

A menudo los pacientes muestran un mal control de sus sentimientos llegando a la agresividad tanto verbal como física, poseyendo una conducta aberrante o se pueden mostrar con un miedo intenso o pánico excesivo ante las situaciones desconocidas, siendo así un verdadero reto para el profesional manejar a estos pacientes. ${ }^{6}$

Son muy comunes las estereotipias motoras, incluyen el aleteo de manos, movimientos constantes de la cabeza, así como el balanceo de sus extremidades o de su cuerpo en general, llegando a mantenerse por largos periodos de tiempo. $^{6}$

A pesar de las condiciones que generalmente se presentan en los pacientes con trastorno autista, no existe una anomalía dental exclusiva.

Habitualmente la caries y la enfermedad periodontal son muy comunes, dado a que la higiene oral es defectuosa puesta por varios factores, tales como la falta de cepillado debido a su condición así como la traba de ellos en aceptar ayuda de otras personas. 6

Hasta el momento no existe ninguna prueba de laboratorio, marcador biológico o un test que diagnostique con certeza el trastorno autista o las enfermedades relacionadas. Según lo expresado, el diagnóstico va estar basado con el juicio clínico del profesional siempre ayudado por los diversos instrumentos de evaluación que confirmen el padecimiento de la patología. 7,8

Aún el tratamiento terapéutico específico para el trastorno autista no existe.

Se utiliza el tratamiento ocasional para el TEA, estos se dividen en farmacológicos y psicopedagógicos, que se tratan de acuerdo a lo que la ocasión lo amerite. ${ }^{9}$

La terapia psicopedagógica desempeña un rol central para el tratamiento de los pacientes con TEA. Actualmente el tratamiento más aceptado es aquel que inicia lo más temprano posible, que sea intensivo y de tipo multidisciplinario, tanto con la terapia de lenguaje, programas de socialización, así como la estimulación auditiva y sensorial, entre otras. ${ }^{9}$

En cuanto al ámbito odontológico el abordaje realizado por el odontopediatra, obliga a realizar modificaciones en los protocolos y estándares que habitualmente se utilizan, dado por la variedad de conductas que puede desencadenar el paciente durante el procedimiento del profesional. Debido a que estos pacientes presentan un mayor riesgo de caries por la poca colaboración, la prevención depende exclusivamente de la habilidad del odontopediatra. ${ }^{10}$

El escenario de atención odontológico es amplio, puede ser ambulatorio en el consultorio dental con el uso de técnicas de desensibilización sistemática, en otros casos el odontopediatra debe coordinarse tanto con los médicos y enfermeras, para una correcta planificación de la atención individualizada del paciente bajo sedación general. ${ }^{2}$

La sedación en el campo de la odontología se considera segura y efectiva cuando es dirigido por el personal capacitado. El paciente debe ser evaluado según su nivel de riesgo previo a la sedación. Su administración es realizada para controlar la conducta y evitar posibles complicaciones que 
se pueden generar a lo largo del tratamiento odontológico. ${ }^{2}$

En los casos de mayor complejidad se recomienda el uso de la anestesia general, proporcionado por la falta de cooperación del paciente a las técnicas mencionadas anteriormente. ${ }^{11}$

\section{PRESENTACIÓN DEL CASO}

Acude a la clínica de Odontopediatría de la División de Estudios de Posgrado e Investigación, de la Facultad de Odontología de la Universidad Nacional Autónoma de México un paciente masculino de 12 años de edad, con diagnóstico médico de Trastorno Autista. Actualmente sus padres refieren cambios conductuales en el paciente como ansiedad y autolesión en la región facial. También han observado un incremento de volumen en la zona retromolar inferior derecha, razón por la cual acude a cita de emergencia, con carácter prioritario de atención. A la exploración extraoral se evaluó el patrón conductual de comportamiento observando rechazo a la inspección, gritos fuertes, imposibilidad de establecer contacto visual y negativismo extremo, clasificando al niño en la escala 1 de Frankl. El paciente es autolesivo constantemente, a tal medida que se mantiene integro con el uso de un casco de protección para evitar traumas en la región facial (Fig. 1).

Fig. 1. Paciente con casco de protección

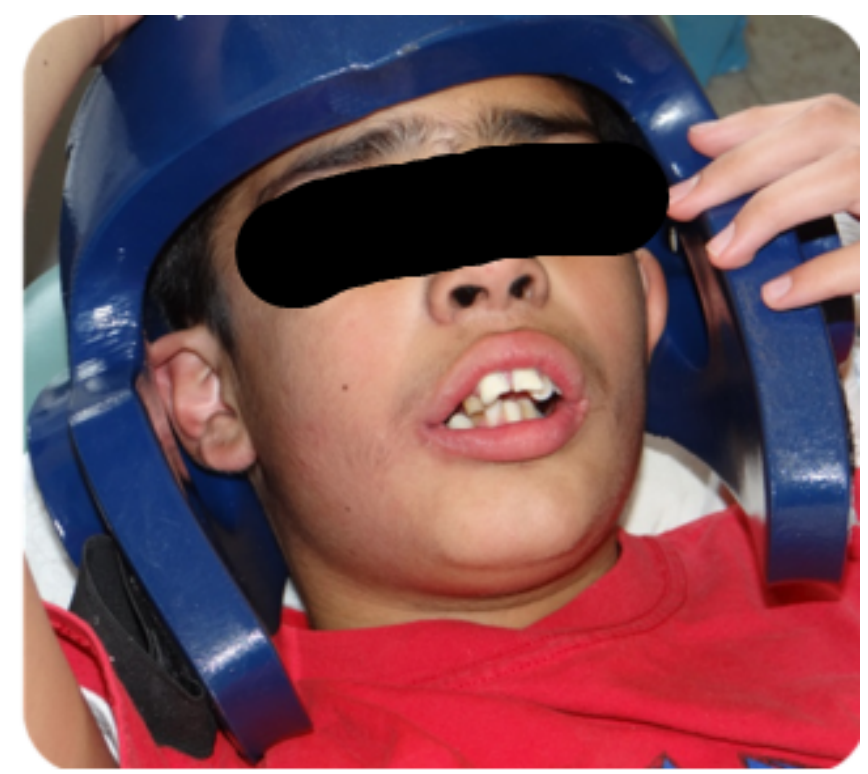

A la exploración intraoral se observa dentición permanente, arcos dentarios colapsados, malposición dentaria, sobremordida vertical y horizontal aumentadas, mordida cruzada posterior del lado izquierdo, abrasión de órganos dentarios 12, 11 y 21 (Fig 2); resinas desajustadas en órganos dentarios 16, 26 (Fig 3), 36 y 46 (Fig 4); hipoplasia en órganos dentarios 17, 15, 14, 24, 25, 27 y gingivitis generalizada. Sin embargo, lo que más llama la atención a la inspección clínica es un aumento de volumen en la zona retromolar inferior del lado izquierdo.

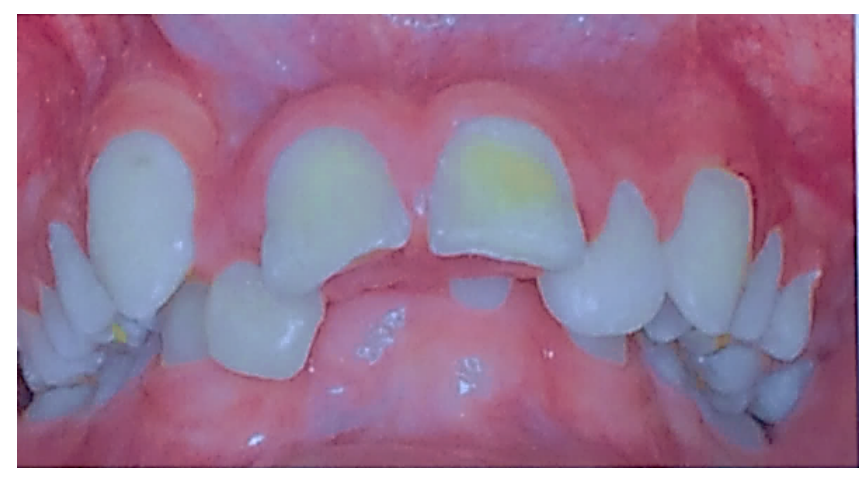

Fig. 2. . Maloclusión con sobremordida

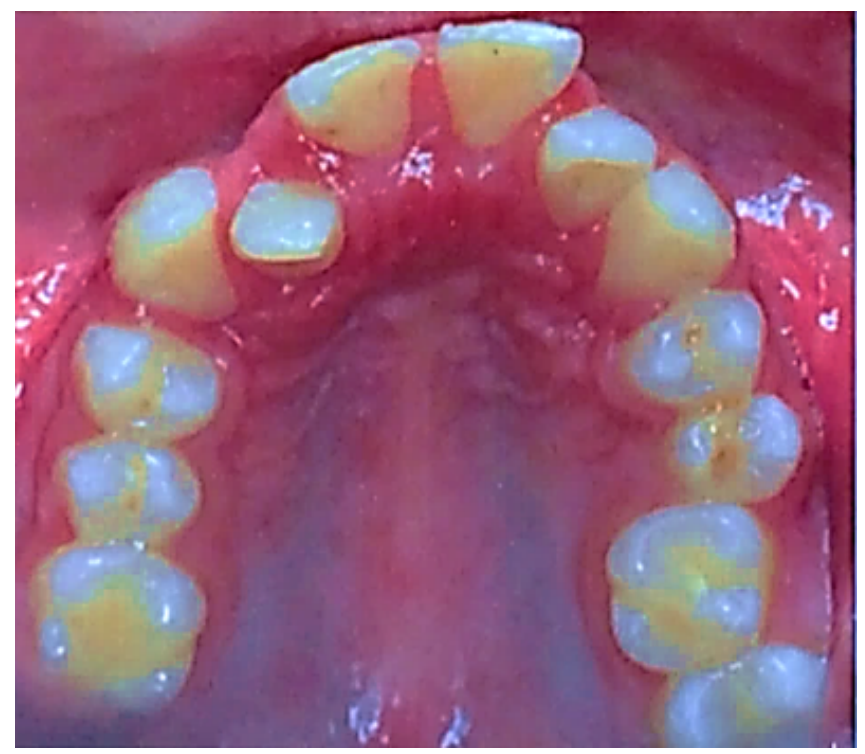

Fig. 3. Muestra el colapso maxilar y restauraciones desajustadas

A la inspección radiográfica se observan dos hallazgos significativos, el primero de ellos es una zona radiopaca en el periápice del órgano dentario 44 (Fig. 5), por lo que se hace interconsulta con el departamento de Patología Bucal y ellos determinan que es compatible con una entidad benigna y que por las características del paciente, se tendrá control radiográfico cada seis meses únicamente. El segundo hallazgo es el órgano dentario 47 (Fig. 6), que se encuentra impactado, se asocia a este diente la inflamación observada clínicamente.

En la radiografía panorámica (por obvias razones se encuentra distorsionada) se puede apreciar el caso más ampliamente, se observa que ambos segundos molares inferiores están impactados y la presencia de los órganos dentarios 18 y 28 (Fig. 7), por lo que se hace interconsulta con el departamento de Cirugía Maxilofacial y se determina la necesidad 


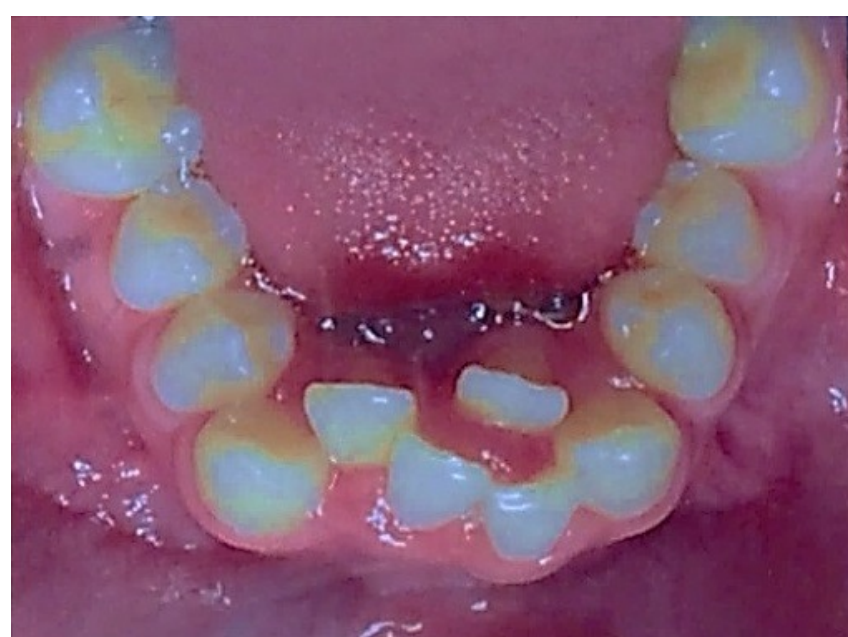

Fig. 4. Arcada inferior que muestra apiñamiento

de realizar las 4 cirugías correspondientes.

Tras realizar la evaluación odontológica se decide que dada la condición bucal y sistémica del niño, la necesidad de procedimientos quirúrgicos y la amplia demanda de maniobras restauradoras, lo idóneo es realizar los tratamientos bajo anestesia general. Una vez que se llegó al acuerdo verbal y escrito bajo consentimiento informado con los padres del paciente, se solicitó a los mismos acudan con su hijo a una valoración pre- anestésica con el médico anestesiólogo. El anestesiólogo valoró el caso y los exámenes de laboratorio, determinando que el paciente presenta condiciones de salud general y se explicó a los padres las indicaciones preoperatorias. De este modo, se programó el procedimiento para anestesia general. Se trata al paciente mediante anestesia general endovenosa, haciendo la fase de inducción con sevoflurano, una vez que se llegó a un plano profundo de hipnosis, se realizó la intubación. Tras la inducción se administró midazolam y se mantuvo la anestesia con sevoflurano y ventilación asistida con una mezcla de oxígeno y aire mediante intubación nasotraqueal. Se empleó monitorización mediante ECG, presión arterial y saturación arterial de oxígeno.

El procedimiento de rehabilitación odontológica interdisciplinaria fue llevado a cabo en tres tratamientos:

1) Tratamiento restaurador: El cual consistió en la rehabilitación de los órganos dentarios que presentaban restauraciones defectuosas, zonas hipoplasicas y abrasión. Se comienza colocando solución anestésica de lidocaína con epinefrina al 2\% 1:100.000 y aislamiento absoluto con dique de hule previo a la fase operatoria en cada uno de los cuadrantes. Restauraciones de Ionómero de vidrio tipo II Ketac Molar $\AA$ en órganos dentarios 17, $15,14,24,25,27$. Se pulen las superficies dentales para eliminar la placa dentobacteriana de las zonas cavitadas, se evalúa la presencia de caries, siendo negativa. Se secan la superficies dentales, se graba con ácido poliacrílico

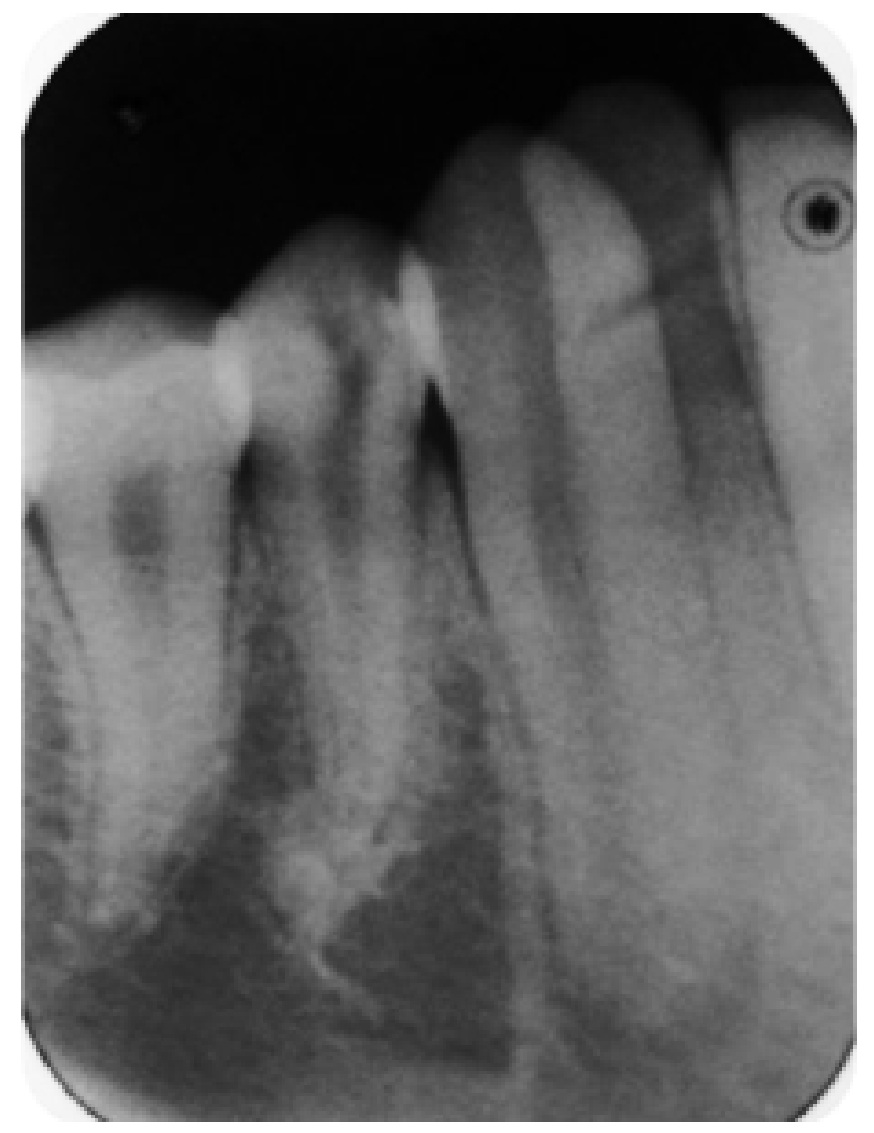

Fig. 5. Radiografía periapical con zona radiopaca en 44

durante 20 segundos, se lava abundantemente para después secar nuevamente la superficie haciéndola apta para colocar el material. Se coloca el ionomero adaptándolo perfectamente a la superficie dentaria. Se recubre con adhesivo y se polimeriza por 10 segundos.

Restauraciones de amalgama en órganos dentarios 16 , 26,36 y 46 . Se eliminan las resinas desajustadas presentes, teniendo especial cuidado en realizar cavidades ideales para recibir restauraciones con amalgama. Se coloca un protector pulpar a base de hidróxido de calcio y se procede a condensar el material y a darle anatomía según el diente y a dejar una superficie tersa (Fig. 9).

Restauraciones interinas con Ionómero de vidrio modificado con resina Ketac $\mathrm{N} 100 \AA$ en los órganos dentarios 12,11 y 21 . Se secan la superficies dentales, se graba con ácido poliacrílico durante 20 segundos, se lava abundantemente para después secar nuevamente la superficie haciéndola apta para colocar el primer y polimerizar durante 10 segundos. Posteriormente se adapta el material prestando especial cuidado en dar una forma lo más adecuada y estética posible, polimerizando 20 segundos. Se da terminación y pulido fino a las restauraciones (Fig $10)$. 

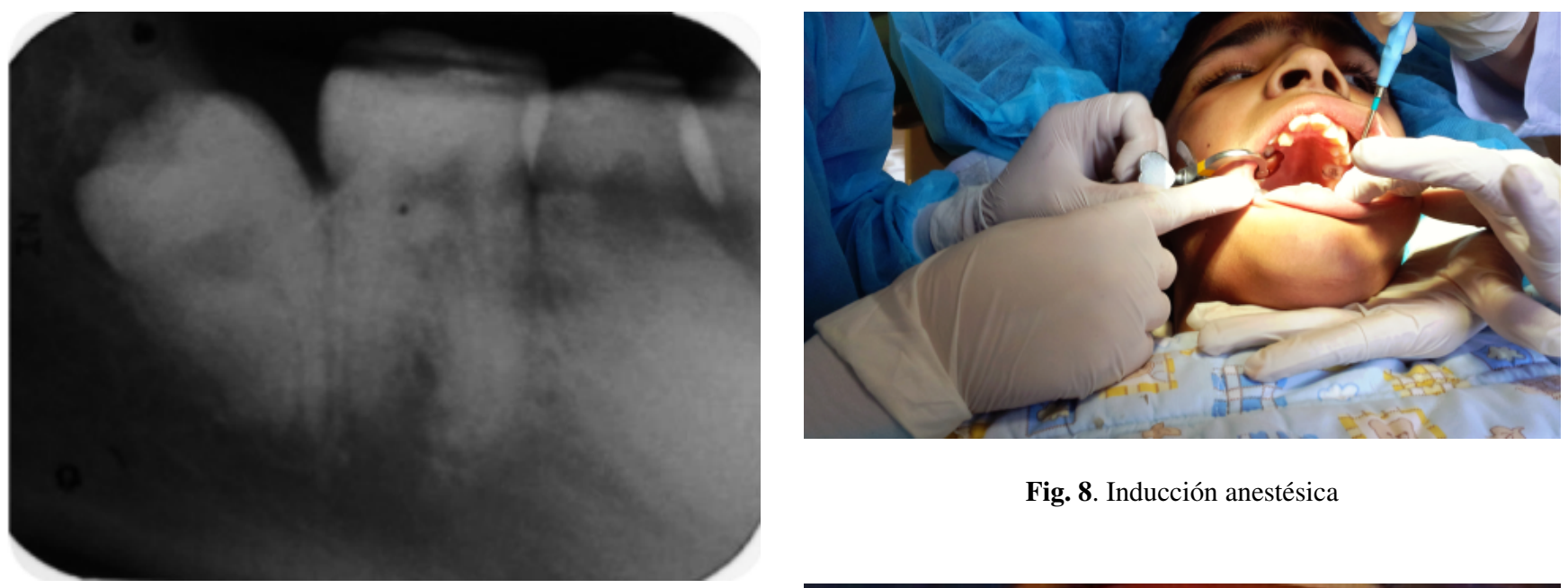

Fig. 8. Inducción anestésica

Fig. 6. Diente 47 impactado

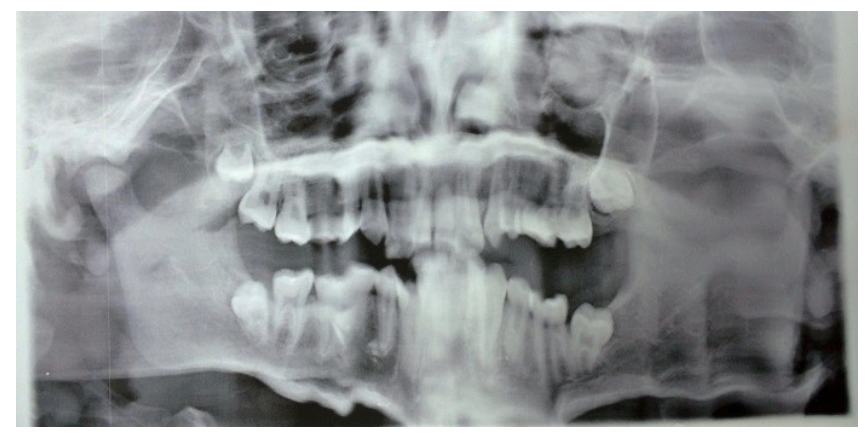

Fig. 7. Radiografía panorámica que muestra retenciones dentarias

Selladores de fosetas y fisuras en los órganos dentarios $35,34,44$ y 45 . Previo pulido de la superficie dental para eliminar la placa dentobacteriana presente. Se grabó con ácido fosfórico (H3PO4) al $37 \%$ durante 15 segundos, se lava, se seca y se colocó el sellador en las superficies oclusales (Fig 11).

2) Tratamiento Quirúrgico

Con el uso de campos estériles y tras llevar a cabo la asepsia y antisepsia del área quirúrgica, se administró anestesia local a base de lidocaína y epinefrina al $2 \%$ 1:100.000 en la región mandibular y maxilar. Se realizaron dos incisiones tipo semi Newman para odontectomias de los OD 37 y 47, posterior a ello se procedió a la desperiostización de tejidos y levantamiento de colgajo de espesor total, se llevó a cabo la osteotomía periférica y luxación a través de elevadores rectos, para conseguir la avulsión de los órganos dentarios (Fig. 12). De inmediato se continuó con el curetaje, irrigación, lavado de lecho quirúrgico y reposición de tejidos usando vicryl 3-0 para control de hemostasia. En región maxilar se realizó el mismo procedimiento y técnica quirúrgica, la incisión

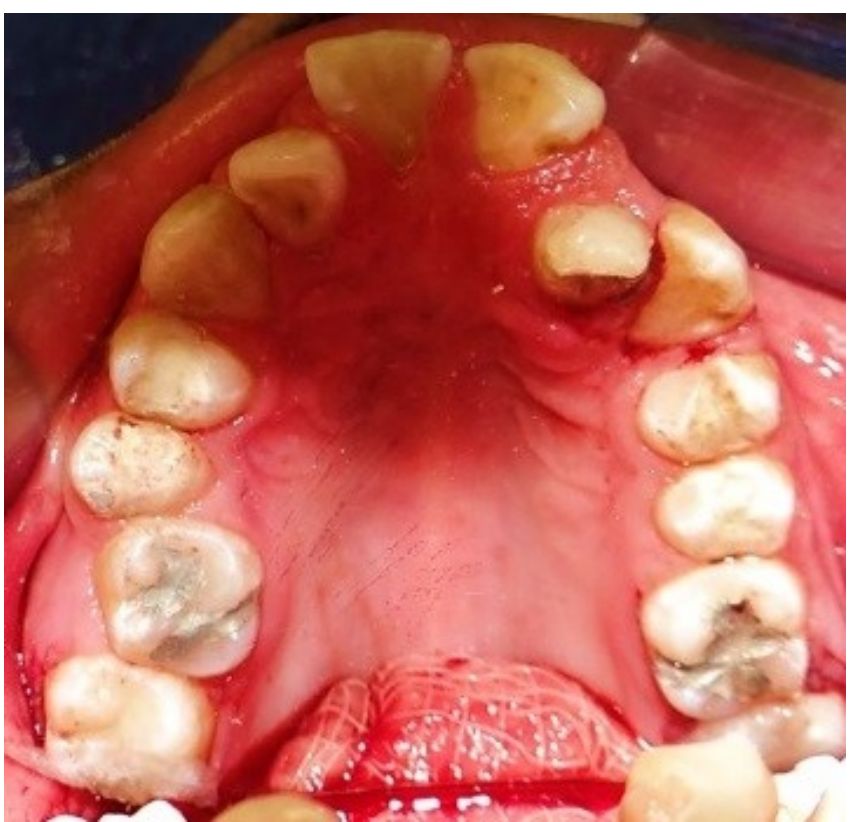

Fig. 9. Fase restauradora completa en la arcada superior

realizada fue de tipo semi Newman, se levantaron colgajos de espesor total, osteotomía, luxación y avulsión de órganos dentarios 18 y 28, posteriormente irrigación y lavado de lecho quirúrgico con reposición de tejidos y uso de vicryl 3-0 para control de hemostasia.

El procedimiento duró 180 minutos, el paciente se mantuvo estable en todo momento, sin incidencias intraoperatorias. Se retiró el taponamiento realizado con gasa y se aspiró el contenido gástrico introduciendo una sonda nasogástrica muy suavemente. El proceso de despertar del paciente fue rápido, retirando el tubo nasotraqueal cuando el nivel de consciencia del paciente fue adecuado. Se trasladó a la unidad de recuperación postanestesia donde permaneció 30 minutos y se permitió la compañía de los padres. El paciente fue dado de alta domiciliaria 


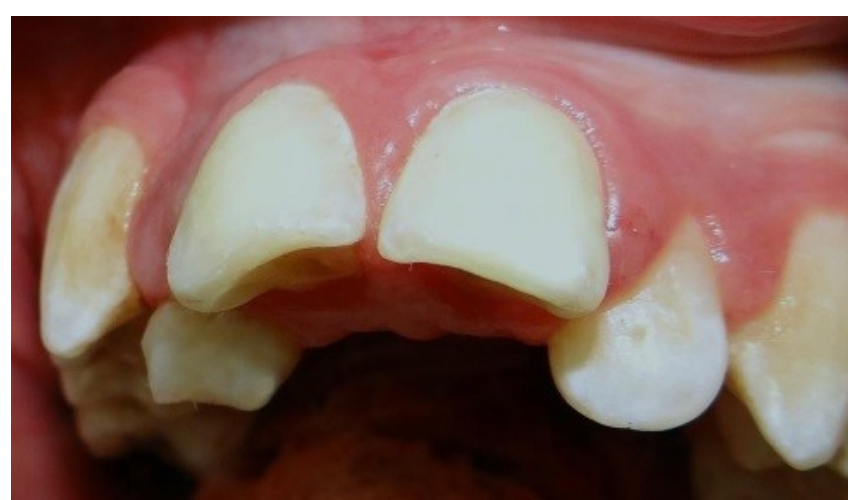

Fig. 10. Restauraciones interinas

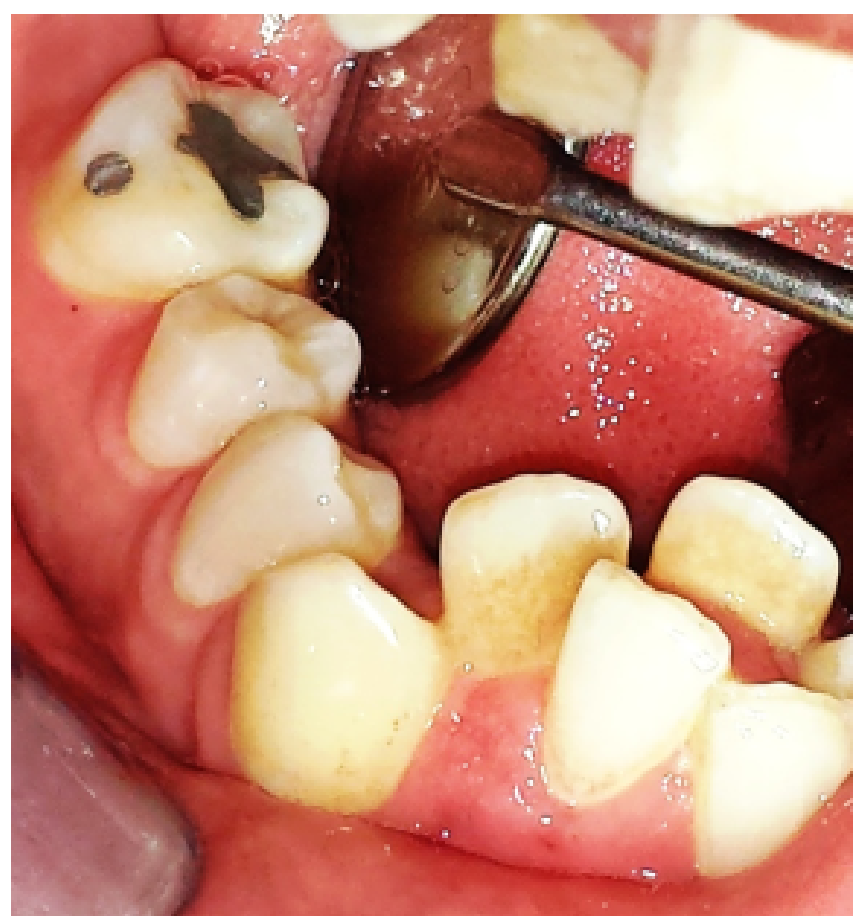

Fig. 11. Tratamiento restaurador del cuarto cuadrante

ese mismo día sin complicaciones. Se indicó a los padres establecer medidas de higiene y control de dieta. Se dieron citas de revisión a una semana, un mes y cuatro meses después del procedimiento sin ninguna eventualidad y con muy buenos resultados.

3) Se acordó con los padres programar citas de control en la clínica de Odontopediatría, con el empleo de técnicas conductuales de restricción física y desensibilización, dichas citas se llevaron a cabo cada cuatro meses (Fig. 13), realizando los siguientes procedimientos preventivos: Control personal de placa bacteriana, profilaxis dental, aplicación tópica de fluoruro de sodio al $5 \%$ Clinproß White Varnish, refuerzo de técnica de cepillado con

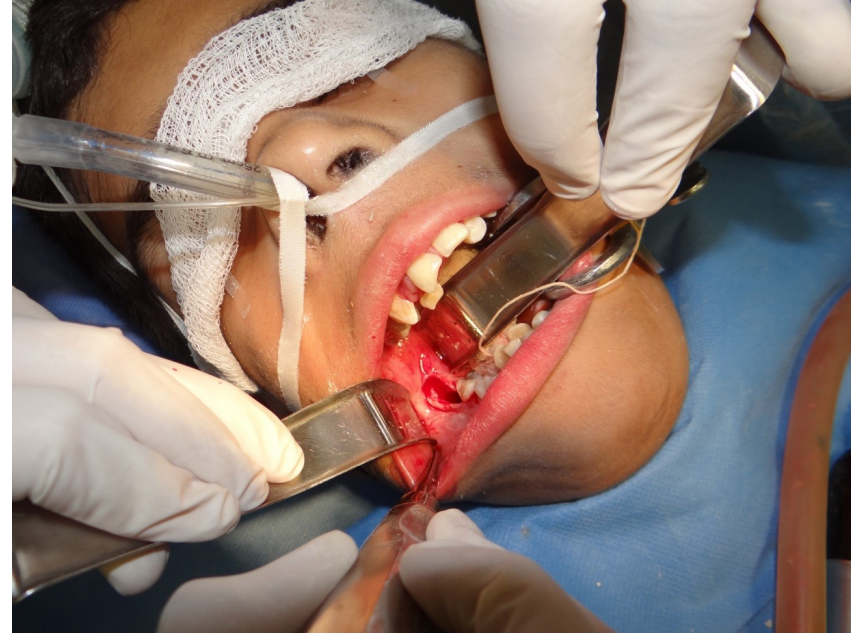

Fig. 12. Tratamiento quirúrgico

los padres y el niño, recomendaciones dietéticas sobre alimentos cariogénicos.

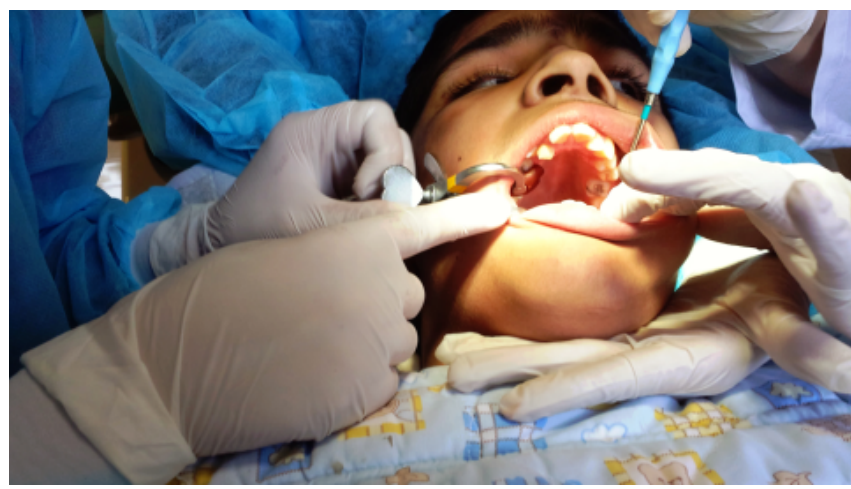

Fig. 13. Fase de mantenimiento

\section{DISCUSIÓN}

Casanova y Acuña, ${ }^{9}$ mostraron que la anestesia general y la sedación intravenosa son utilizadas cuando se requiere que el paciente se mantenga inmóvil por un lapso de tiempo prolongado en el consultorio odontológico. Es frecuente el uso de la anestesia general en aquellos pacientes difíciles que por su propia condición no pueden seguir las indicaciones que se les plantea.

De Nova et al. ${ }^{10}$ recomendó el uso de anestesia general en tratamientos dentales a los niños con diversidades funcionales como el caso expuesto de TEA, pero no es necesario la misma intervención en todos los casos.

Basados en esto, en este estudio el paciente con TEA fue manejado multidisciplinariamente y bajo un esquema de 
anestesia general por la dificultad para establecer comunicación, la falta de cooperación y los procedimientos dentales amplios requeridos por el paciente.

Se manejó sistema de aislamiento para tener el mayor control de fluidos y al mismo tiempo para la mejor manipulación de los materiales restauradores utilizados. De acuerdo a Antonio et al. ${ }^{2}$ la técnica restrictiva se emplea en situaciones complejas, teniendo en cuenta el mayor número de sesiones que requieren estos pacientes.

Por lo anterior, las citas de control se realizaron con restricción física por la rapidez de los procedimientos programados y con la presencia de los padres que ayudaron en todo momento a establecer una mejor comunicación con el paciente.

De acuerdo con Gómez et al., ${ }^{3}$ se debe emplear un protocolo de desensibilización sistemática por medio de imagines o videos que se pueden combinar previo a la consulta dental, facilitando el procedimiento odontológico.

Atienza $^{11}$ enfatiza el uso de pictogramas como apoyos visuales. Las personas con TEA comprenden, asimilan y retienen mejor la información que se les presenta de manera visual.

Según Antonio et al., ${ }^{2}$ el uso de imágenes en lugar de palabras permite una mayor cooperación en el tratamiento dental, ya que facilita la familiarización con el proceso odontológico.

Tomando en cuenta esto último, se planifica que en las citas de control se ponga en práctica el uso de pictogramas antes y durante la consulta para ayudar a establecer una mejor comunicación y a mejorar la conducta del paciente durante la consulta.

\section{CONCLUSIONES}

El profesional que trate con pacientes con TEA debería conocer las características del síndrome con la finalidad de dar un mejor tratamiento. Los pacientes con TEA pueden presentar mayor riesgo de patología oral debido a su capacidad disminuida de participar en la higiene diaria, la presencia de hábitos parafuncionales y autolesión. Las personas con TEA pueden padecer ciertos grados de discapacidad intelectual y de control motor, cuestión que debe ser tomada en cuenta en el manejo conductual. Para el mantenimiento de la salud bucal es necesaria la colaboración de los padres enfatizando la importancia de las revisiones periódicas, una buena técnica de cepillado y consejo dietético. El odontólogo se verá beneficiado al desarrollar estrategias para manejar a los pacientes con TEA asegurando un tratamiento efectivo. Se debe emplear un método diferente en relación a las características de cada paciente sin modificar los métodos convencionales de tratamiento odontológico.

Conflicto de intereses y financiamiento Los autores declaran no tener conflicto de intereses, haber cumplido con los requisitos de autoría y haber autofinanciado este artículo.

\section{Referencias}

1 Guía de Práctica Clínica para el Manejo de Pacientes con Trastornos del Espectro Autista en Atención Primaria. 2009.

2 Antonio F, Mill E, Manejo de Pacientes con Diversidad Funcional en el Ámbito Odontológico, Revista Venezolana de Investigación Odontológica. 2013; 1 (2): 121-135.

3 Gómez B, Badillo V, Intervención odontológica actual en niños con autismo. La desensibilización sistemática, Cient Dent. 2009;6;3:207-215.

4 Fuentes J, Guía de buena práctica para el tratamiento de los trastornos del espectro autista, Revista de Neurología APNA. 2006; 43 (7): 425-438.

5 Cabanyes J, García E, Identificación y diagnóstico precoz de los trastornos del espectro autista, Revista de Neurología. 2004; 39 (1): 81-90

6 Rogel FJ, Autismo (Revisión Bibliográfica), Gaceta Médica de México. 2005; 2: 142-147

7 Etchepareborda MC, Perfiles Neurocognitivos del Espectro Autista, Revista de Neurología Clínica. 2001; 2:175-192

8 León MM, Paciente autista en el ámbito odontológico: autoagresiones versus maltrato infantil, Gaceta Internacional de Ciencia Forense. 2013; 9: 2174-9019

9 Casanova J, Acuña G. Discapacidad en el paciente odontológico, Revista Mexicana de Odontología Clínica. 2006; 1(6-7): 30-32.

10 De Nova J, López N, Criteria for selecting children with special needs for dental treatment under general anaesthesia, Rev. Med Oral Patol Oral Cir Bucal 2007; 12(7): 496-503.

1 Atienza O, Manejo de Pacientes con TEA en Odontología, Práctica Clínica 2014; 261:176-201.

Recibido: 1 de Diciembre de 2016

Aceptado: 30 de Diciembre de 2016 
